\title{
Attribute-Based Bandwidth Reservation Scheme for Mobile Multimedia Wireless Networks
}

\author{
Jau-Yang Chang ${ }^{\S}{ }^{\dagger}$ and Hsing-Lung Chen ${ }^{\dagger}$ \\ ${ }^{\S}$ Department of Electronic Engineering \\ Chinmin Institute of Technology \\ Toufen, Taiwan, R.O.C. \\ ${ }^{\dagger}$ Department of Electronic Engineering \\ National Taiwan University of Science and Technology \\ Taipei, Taiwan, R.O.C. \\ jychang@ms.chinmin.edu.tw,hlchen@et.ntust.edu.tw
}

\begin{abstract}
In order to provide seamless multimedia services in the next-generation wireless networks, efficient bandwidth reservation scheme must be developed. A novel attributebased bandwidth reservation scheme is proposed in this paper as a solution to support quality-of-service guarantees $(o S)$ in the mobile multimedia wireless networks. Based on the existing network conditions, the proposed scheme makes an adaptive decision for bandwidth reservation and call admission in each base station (BS). We use the dynamically adaptive approaches to reduce the connection-blocking probability $(C B P)$ and connectiondropping probability (CDP), and to increase the bandwidth utilization (BU) for mobile multimedia wireless networks. Simulation results show that our proposed scheme outperform the previously proposed scheme in terms of $C B P, C D P$, and $B U$ in mobile communication systems.
\end{abstract}

\section{Introduction}

Providing multimedia services with a QoS guarantee in mobile wireless networks presents more challenges due to user's mobility and limited bandwidth resource. Since bandwidth is the most critical resource in mobile multimedia wireless networks, it is important to employ mechanisms for efficiently using the available bandwidth. In mobile cellular communication networks carrying multimedia traffic, it becomes necessary to provide QoS guarantees for multimedia traffic connections. In order to utilize the radio spectrum efficiently, microcellular and picocellular networks are deployed. These networks have the inherent problem of rapid handoffs due to smaller coverage areas of BSs. This problem leads to higher CDP and results in bandwidth resource availability varying repeatedly. Frequent changes in the network traffic make the provision of guaranteed QoS more difficult. Reserving bandwidth for handoff mobile hosts (MHs) can reduce CDP in wireless networks. However, it may lead to high CBP for new connections and low BU. Hence, research in the area of QoS provisioning in the next-generation wireless networks focuses on the integration of resource reservation and admission control policies [1]-[6].

Recently, several bandwidth reservation schemes have been proposed to support QoS provisioning in wireless environments. In [2], the shadow cluster concept is used to predict future resource allocation and to perform bandwidth reservation in wireless networks. In this scheme, the home BS of the MH informs the BSs of its shadow cluster area about the MH's requirement, position, and movement parameters. It requires the exchange of a large amount of detailed knowledge of MHs between the BSs. This scheme increases too many overheads between the BSs, especially in microcellular and picocellular wireless systems. Moreover, it is difficult to accurately estimate future movement patterns in real wireless environments. In [3], the adaptive bandwidth reservation scheme provides QoS guarantees by reserving bandwidth in all neighboring cells. In this scheme, if any reservation in neighboring cells fails, the new connection and handoff connection are rejected. It increases CBP and CDP. Moreover, bandwidth is reserved redundantly because the $\mathrm{MH}$ moves to only one of neighboring cells. It decreases the BU. An adaptive resource allocation for multimedia QoS management in wireless networks is proposed in [1] to improve CBP, CDP, and BU by using the dynamic guard-channel scheme. However, this scheme does not appropriately adjust the reserved bandwidth for each BS in bursts of traffic conditions. It reduces the BU and increases the CDP.

This paper introduces a novel attribute-based bandwidth reservation scheme that supports QoS guarantees in the next generation mobile multimedia wireless networks. In the proposed scheme, each BS makes an adaptive decision for bandwidth reservation by employing attributemeasurement mechanism and traffic-based reservation strategy. The amount of reserved bandwidth for each BS is dynamically adjusted, according to the on-line traffic in- 
formation of each BS. The traffic-based reservation strategy makes the system more responsive to the current traffic conditions, providing efficient bandwidth reservation for the handoff connections. The control of bandwidth reservation is adaptive under overloading traffic conditions, thus can effectively deal with sudden traffic surges. Multimedia traffic is a combination of both real-time and nonreal-time traffic. Real-time traffic includes voice and video while data and graphics make up nonereal-time traffic. We design efficient call admission control algorithms for real-time and nonreal-time traffic, according to the different multimedia services. On-line bandwidth reservation scheme does not require prediction of future traffic intensity and user mobility. This approach has significantly lower complexity and system overhead than mobility oriented methods. Our proposed scheme is an adaptive and on-line mechanism. It only requires minimal computational overhead in each BS, and no communication overhead between neighboring BSs to request and release reservations.

The remainder of this paper is organized as follows. Section 2 describes our assumed model of wireless networks. In Section 3, we illustrate the proposed scheme in detail. In Section 4, we present our simulation model and analyze the comparative evaluation results of the proposed scheme through simulations. Finally, Section 5 offers some concluding remarks.

\section{System Model}

We consider a multimedia mobile wireless network with a cellular network infrastructure. Each cell contains a BS that needs to allocate and reserve bandwidth for MHs, and communicates with other BSs. BSs are connected to a switching network through wired lines. Two types of connections share the bandwidth of the BS for each cell: new connections and handoff connections. A BS in a cellular network may receive new connections of MHs within its cell and handoff connections of MHs from the neighboring cells. When a connection arrives at a cell where the bandwidth is not available, this situation is called connection blocking for new connections or connection dropping for handoff connections. For a mobile user, dropping an ongoing connection is generally more unacceptable than blocking a new connection request. Therefore, reducing the CDP is usually a main objective in the mobile wireless communication systems. The connection blocking rate and connection dropping rate are two most significant connection-level QoS parameters in the mobile wireless networks [1], [5], [6].

In the proposed scheme, it is assumed that when an $\mathrm{MH}$ requests a new connection in the current cell or moves into the neighboring cell, the following parameters are provided: 1) The traffic class (I or II). 2) The required bandwidth for the connection. 3) The minimum required bandwidth for the connection. The minimum required bandwidth connection is the smallest amount of bandwidth that the connection can be assured of a certain acceptable QoS, e.g., the smallest encoding rate of its codec [3].

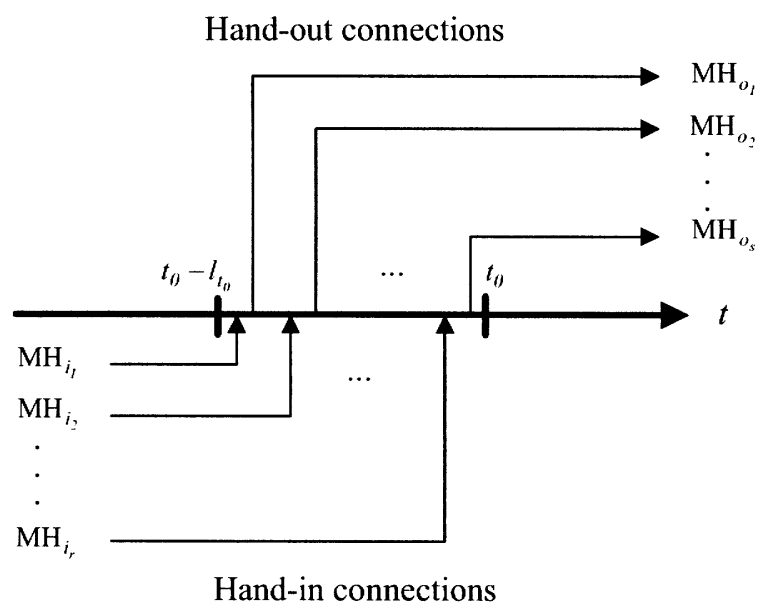

Figure 1. Hand-in and hand-out connections within the time interval from $\quad l_{t_{0}}$ to in the cell .

\section{Proposed Bandwidth Reservation Scheme}

Bandwidth reservation is one of the critical elements in ensuring the QoS in mobile wireless networks and thus, requires mechanisms to efficiently use the available bandwidth. The proposed scheme combines attributemeasurement mechanism, traffic-based reservation strategy, and call admission control algorithms to guarantee QoS requirements in mobile wireless environments. The attribute-measurement mechanism is employed to measure the traffic information. The traffic-based reservation strategy is employed to calculate the amount of reserved bandwidth. The call admission control algorithms are employed to control whether the connections can be served or not. These mechanisms are executed at each BS in a distributed manner.

\subsection{Attribute-measurement mechanism}

In order to implement the attribute-measurement mechanism, we monitor several traffic parameters within each time interval in the cell. In the proposed scheme, we classify all data traffic into real-time (Class I) and nonrealtime (Class II) according to the different multimedia services. We define the hand-in and hand-out connections for Class I (or Class II) data traffic within each time interval in the cell. The connection for an incoming handoff $\mathrm{MH}$ in the cell is called hand-in connection and the connection for an outgoing handoff $\mathrm{MH}$ in the cell is called hand-out connection. Figure 1 shows the hand-in and hand-out connections within the time interval from $t_{0}-l_{t_{0}}$ to $t_{0}$ in cell $j$, where $t_{0}$ is the current time and $l_{t_{0}}$ is the length of time interval at time $t_{0}$, and $\mathrm{MH}_{i_{r}}$ denotes the rth hand-in connection and $\mathrm{MH}_{o}$ denotes the sth hand-out connection. Let $B I_{t, j}^{k}$ and $B O_{t, j}^{k}$, respectively, be the sum of required bandwidths of hand-in connections and the sum of released bandwidths of hand-out connections for the class $k$ data traffic at time $t$ in cell $j$, where $t$ is measured time from $t_{n}-l_{t_{n}, j}^{k}$ to $t_{n}$. The $l_{t_{n}, j}^{k}$ 
denotes the length of time interval for class $k$ data traffic at time $t_{n}$ in cell $j$. Let $\alpha_{t_{n}, j}^{k}$ denote the bandwidth rate of hand-in and hand-out connections for class $k$ data traffic at time $t_{n}$ in cell $j$. The $\alpha_{t_{n}, j}^{k}$ can be expressed by

$$
\alpha_{t_{n}, j}^{k}=\frac{\sum_{t=t_{n}-l_{t_{n}, j}^{k}}^{t_{n}} B I_{t, j}^{k}-\sum_{t=t_{n}-l_{t_{n, j}}^{k}}^{t_{n}} B O_{t, j}^{k}}{\max \cdot\left\{\sum_{t=t_{n}-l_{t_{n}, j}^{k}}^{t_{n}} B I_{t, j}^{k}, \sum_{t=t_{n}-l_{t_{n, j}^{k}}^{k}}^{t_{n}} B O_{t, j}^{k}\right\}} .
$$

The parameter $\alpha_{t_{n, j}}^{k}$ represents bandwidth variation of MHs moving into and departing from the cell $j$ for the class $k$ data traffic within the time interval $\left[t_{n}-l_{t_{n}, j}^{k}, t_{n}\right]$. If $\alpha_{t_{n}, j}^{k}$ is a positive value, it means that the sum of required bandwidths of hand-in MHs is larger than the sum of released bandwidths of hand-out MHs for the class $k$ data traffic within the time interval $\left[t_{n}-l_{t_{n}, j}^{k}, t_{n}\right]$ in cell $j$.

Let $H I_{t, j}^{k}$ and $H O_{t, j}^{k}$, respectively, be the number of hand-in connections and the number of hand-out connections for the class $k$ data traffic at time $t$ in cell $j$. Let $\beta_{t_{n}, j}^{k}$ denote the numeral rate of hand-in and hand-out connections for class $k$ data traffic at time $t_{n}$ in cell $j$. The $\beta_{t_{n}, j}^{k}$ can be expressed by

$$
\beta_{t_{n}, j}^{k}=\frac{\sum_{t=t_{n}-l_{t_{n}, j}^{k}}^{t_{n}} H I_{t, j}^{k}-\sum_{t=t_{n}-l_{t_{n}, j}^{k}}^{t_{n}} H O_{t, j}^{k}}{\max \cdot\left\{\sum_{t=t_{n}-l_{t_{n}, j}^{k}}^{t_{n}} H I_{t, j}^{k}, \sum_{t=t_{n}-l_{t_{n}, j}^{k}}^{t_{n}} H O_{t, j}^{k}\right\}} .
$$

The parameter $\beta_{t_{n}, j}^{k}$ represents variation of MHs moving into and departing from the cell $j$ within the time interval $\left[t_{n}-l_{t_{n}, j}^{k}, t_{n}\right]$. If $\beta_{t_{n}, j}^{k}$ is a negative value, it means that the number of hand-out MHs is larger than the number of hand-in MHs within the time interval $\left[t_{n}-l_{t_{n}, j}^{k}, t_{n}\right]$ in cell $j$.

Let $D I_{t, j}^{k}$ denote the number of dropping connections for the class $k$ data traffic at time $t$ in cell $j$. Let $\gamma_{t_{n}, j}^{k}$ be the CDP of class $k$ data traffic at time $t_{n}$ in cell $j$, which can be expressed by

$$
\gamma_{t_{n}, j}^{k}=\frac{\sum_{t=t_{n}-l_{t_{n}, j}^{k}}^{t_{n}} D I_{t, j}^{k}}{\sum_{t=t_{n}-l_{t_{n}, j}^{k}}^{t_{n}} H I_{t, j}^{k}} .
$$

In order to make the system responsive to the bursts of traffic conditions, the length of time interval $\left[t_{n}-l_{t_{n}, j}^{k}, t_{n}\right]$ is chosen based on the variation of CDP for each time interval. Let $\delta_{t_{n}, j}^{k}$ denote variation of $\gamma_{t_{n-1}, j}^{k}$ and $\gamma_{t_{n}, j}^{k}$ for class $k$ data traffic at time $t_{n}$ in cell $j$, which can be expressed by

$$
\delta_{t_{n}, j}^{k}=\frac{\gamma_{t_{n}, j}^{k}}{\gamma_{t_{n-1}, j}^{k}} .
$$

The length of time interval $\left[t_{n}-l_{t_{n}, j}^{k}, t_{n}\right]$ in cell $j$ is dynamically adjusted according to $\delta_{t_{n}, j}^{k}$. The value of $l_{t_{n}, j}^{k}$ can be calculated by

$$
l_{t_{n}, j}^{k}=l_{t_{n-l}, j}^{k}+\rho_{t_{n}, j}^{k} \cdot u,
$$

where $u$ is an unit time and $\rho_{t_{n}, j}^{k}$ is given by

$$
\rho_{t_{n}, j}^{k}=\left\{\begin{array}{cc}
{\left[\delta_{t_{n}, j}^{k}\right]} & \text { if } \delta_{t_{n}, j}^{k} \geq 1 \\
\left.-\frac{1}{\delta_{t_{n}, j}^{k}}\right] & \text { if } 0<\delta_{t_{n}, j}^{k}<1 . \\
0 & \text { if } \delta_{t_{n}, j}^{k}=0
\end{array} .\right.
$$

The $\alpha_{t_{n}, j}^{k}, \beta_{t_{n}, j}^{k}$ and $\gamma_{t_{n}, j}^{k}$ highly affect the operation of a reservation system in mobile communication networks. We define $\alpha_{t_{n}, j}^{k}, \beta_{t_{n}, j}^{k}$, and $\gamma_{t_{n}, j}^{k}$ as reservation-factors for class $k$ data traffic at time $t_{n}$ in cell $j$. When $\alpha_{t_{n}, j}^{k}>0$ (or $<0$ ) and $\beta_{t_{n}, j}^{k}>0$ (or $>0$ ), it indicates under the heavy (or light) traffic load for class $k$ data traffic at time $t_{n}$ in cell $j$. On the other hand, When $\alpha_{t_{n}, j}^{k}>0$ and $\beta_{t_{n}, j}^{k}<0$, or $\alpha_{t_{n}, j}^{k}<0$ and $\beta_{t_{n}, j}^{k}>0$, it represents less traffic fluctuations for class $k$ data traffic at time $t_{n}$ in cell $j$. According to $\gamma_{t_{n}, j}^{k}, \delta_{t_{n}, j}^{k}$ can be calculated which represents the variation of CDP for class $k$ data traffic at time $t_{n}$ in cell $j$. When $\delta_{t_{n}, j}^{k}>1$ (or $<1$ ), it means that the CDP for class $k$ data traffic is an increased (or decreased) trend at time $t_{n}$ in cell $j$. Hence, for efficiently adjusted the reservationfactors and bandwidth reservation, the value of $l_{t_{n}, j}^{k}$ should be increased (or decreased). The initial value of $l_{t_{n}, j}^{k}$ and $u$ may be chosen based on the real operation of system (e.g., $l_{t_{n}, j}^{k}=60 \mathrm{sec}$ and $u=10 \mathrm{sec}$ ). The reservation-factors bring an immediate response on the traffic conditions. They not only make the system responsive to the bursts of traffic conditions, but also get the bandwidth reservation more efficiently.

\subsection{Traffic-based reservation strategy}

The traffic-based reservation strategy is employed to calculate the amount of reserved bandwidth based on the reservation-factors and network conditions. The basic idea of this strategy is that a reservation system with lower complexity does not require advance knowledge or prediction of future traffic intensity and user mobility. This strategy makes lower system overhead and causes control decisions of bandwidth reservation in real time. In this framework, the BSs record the requested bandwidths of MHs within each time interval for each traffic class. Let $R_{j_{n+1}}^{k}$ denote the amount of new reserved bandwidth for the next time and $R_{j_{n}}^{k}$ the amount of reserved bandwidth at the current time $t_{0}$ for the class $k$ data traffic in the BS of cell $j$. Let $B_{w, j}^{k}$ is the amount of hand-in requested bandwidths 
for class $k$ data traffic within the time interval $w$ in the BS of cell $j$, which can be expressed by

$$
B_{w, j}^{k}=\sum_{t=t_{n}-l_{t o, j}^{k}}^{t_{n}} B I_{t, j}^{k} .
$$

Then, the $R_{j_{n+1}}^{k}$ can be calculated by

$$
R_{j_{n+1}}^{k}=\left(\frac{1-\varepsilon_{j}^{k}}{2}\right) R_{j_{n}}^{k}+\left(\frac{1+\varepsilon_{j}^{k}}{2}\right) B_{w . j}^{k},
$$

where $\varepsilon_{j}^{k}$ is the explicit reservation-factor which can be calculated by

$$
\varepsilon_{j}^{k}=\alpha_{t_{0}, j}^{k}+\beta_{t_{\theta}, j}^{k} .
$$

The explicit reservation-factor controls the relative weights for the recent and past traffic histories. If the mobility of MHs is uniformly distributed, the new reserved bandwidth for the next time in the BS of cell $j$ can rely on the current reserved bandwidth $\left(R_{j_{n}}^{k}\right)$. However, when the traffic distribution is non-uniform, due to the temporal traffic fluctuations, the new reserved bandwidth for the next time in the BS of cell $j$ should depend on recent requested bandwidths of hand-in connections $\left(B_{w, j}^{k}\right)$. We decide the value of $\varepsilon_{j}^{k}$ by considering the current network traffic conditions. Hence the value of reservation-factor $\varepsilon_{j}^{k}$ can affect the network performance significantly.

For efficient BU, the control decisions of bandwidth reservation must be dynamically adjustable. In our proposed scheme, we take into account the existing network conditions. The amount of reserved bandwidth for each BS is dynamically adjusted, according to the on-line traffic information of each BS. It reserves bandwidth only when necessary. So the BSs make bandwidth reservation efficiently. To provide QoS guarantees, if the reserved bandwidth for the Class I is not sufficient, our scheme allows the use of the bandwidth from the reserved bandwidth for Class II. The traffic-based reservation strategy makes the system more responsive to the current traffic conditions, providing efficient bandwidth reservation for the handoff connections. Therefore, the CDP can be reduced.

\subsection{Call Admission Control Algorithms}

When $\mathrm{MH} x$ requests a new connection in the cell $b j$, the BS of cell $b j$ tries to admit this request by using the available bandwidth. The new connection of MH $x$ is rejected in the cell $b j$ if its required bandwidth is large than the unused bandwidth. Otherwise, the BS of cell $b j$ examines the reservation factors, according to the attributemeasurement mechanism. If there are high reservationfactors (e.g., $\varepsilon_{b j}^{\text {Class }}{ }^{I}+\varepsilon_{b j}^{\text {Class }_{-} I I}>0.6$ ) in the BS of cell $b j$, then the new connection will be blocked. Otherwise, the BS of cell $b j$ accepts the connection and allocates the corresponding bandwidth for the new connection of $\mathrm{MH} x$.

When MH $x$ moves from cell $b j$ to the cell $b k$, the BS of cell $b j$ releases allocated bandwidth of the MH $x$. There are two classes of the call admission control algorithms for handoff connection in the cell $b k$, according to the traffic class of MH $x$. If the handoff connection of $\mathrm{MH} x$ is a realtime data traffic, the BS of cell $b k$ checks whether the available bandwidth plus total reserved bandwidth ( $R_{b k}^{\text {Class }}{ }^{I}+R_{b k}^{\text {Class }}{ }^{I I}$ ) is sufficient or not. If the minimum required bandwidth for handoff connection of $\mathrm{MH} x$ is not sufficient, the BS of cell $b k$ drops the handoff connection of MH $x$. Otherwise, the BS of cell $b k$ accepts the connection and allocates the corresponding bandwidth for the handoff connection of MH $x$.

If the handoff connection of MH $x$ is a nonreal-time data traffic, it is dropped only when there is no residual bandwidth for the $\mathrm{MH} x$ in the BS of cell $b k$. If there is not any available bandwidth and reserved bandwidth $\left(R_{b k}^{\text {Class }}{ }^{I I}\right.$ ) for class II traffic in the BS of cell $b k$, the BS of cell $b k$ drops the handoff connection of MH $x$. Otherwise, the BS of cell $b k$ accepts the connection and allocates the corresponding bandwidth for the handoff connection of $\mathrm{MH} x$.

\section{Performance Analysis}

In this section, we present performance analysis for the proposed scheme. We describe our simulation model and illustrate the simulation results, comparing our scheme with the traditionally non-reserved bandwidth mechanism. The performance of our proposed scheme is also compared an existing adaptive resource allocation scheme [1]. In our simulation model we have the following assumptions.

1) The simulation environment is composed of 100 cells and available bandwidth of each cell is $30 \mathrm{Mb} / \mathrm{s}$, each cell keeping contact with its six neighboring cells. The distance between two BSs is $1 \mathrm{~km}$.

2) The arrival process for new connection requests is Poisson distribution with rate $\lambda$, which is uniform in all cells.

3) Three cases of mobility are considered: MHs move hardly, in which case the speed of MHs are uniformly distributed between 0 and $5 \mathrm{~km} / \mathrm{h}$; MHs move slowly, in which case the speed of MHs are uniformly distributed between 6 and $40 \mathrm{~km} / \mathrm{h}$; and MHs move rapidly, in which case the speed of MHs are uniformly distributed between 41 and $90 \mathrm{~km} / \mathrm{h}$.

4) Six different traffic types are assumed based on bandwidth requirement and traffic class shown in Table 1 [3]

5) The durations of connections are assumed to follow a geometric distribution with different means for different multimedia traffic types [3].

We compare the CBPs, CDPs, and BUs of three mechanisms via simulations. Figure 2 shows the CBP. The CBPs increase in all mechanisms with increase in the arrival rate of new connection request. The unsuitable reservation scheme results in the most of connection blocking. It reserves too many bandwidths for the handoff connection. Our proposed scheme can predict that a little adjustment for bandwidth reservation is enough, resulting in lower CBP. According to the Figure 2, when the connection arri- 
Table 1. Multimedia traffic used in simulation mode

\begin{tabular}{ccccccc}
\hline Application & $\begin{array}{c}\text { Traffic } \\
\text { Class }\end{array}$ & $\begin{array}{c}\text { Bandwidth } \\
\text { Requirement }\end{array}$ & $\begin{array}{c}\text { Average } \\
\text { Bandwidth } \\
\text { Requirement }\end{array}$ & $\begin{array}{c}\text { Connection } \\
\text { Duration }\end{array}$ & $\begin{array}{c}\text { Average } \\
\text { Connection } \\
\text { Duration }\end{array}$ & Example \\
\hline 1 & Real-time & $\begin{array}{c}30 \mathrm{~Kb} / \mathrm{s} \\
(\mathrm{CBR})\end{array}$ & & $60-600$ seconds & 180 seconds & $\begin{array}{c}\text { Voice Service \& Audio- } \\
\text { phone }\end{array}$ \\
\hline 2 & Real-time & $\begin{array}{c}256 \mathrm{~Kb} / \mathrm{s} \\
(\mathrm{CBR})\end{array}$ & $60-1800$ seconds & 300 seconds & $\begin{array}{c}\text { Video-phone \& Video- } \\
\text { conference }\end{array}$ \\
\hline 3 & Real-time & $\begin{array}{c}1-6 \mathrm{Mb} / \mathrm{s} \text { (average) } \\
2.5-9 \mathrm{Mb} / \mathrm{s}(\mathrm{peak}) \\
(\mathrm{VBR})\end{array}$ & $3 \mathrm{Mb} / \mathrm{s}$ & $300-18000$ seconds & 600 seconds & $\begin{array}{c}\text { Interact. Multimedia } \\
\& \text { Video on Demand }\end{array}$ \\
\hline 5 & Nonreal-time & $\begin{array}{c}5-20 \mathrm{~Kb} / \mathrm{s} \\
(\mathrm{UBR})\end{array}$ & $10 \mathrm{~Kb} / \mathrm{s}$ & $10-120$ seconds & 30 seconds & Email, Paging \& Fax \\
\hline 5 & Nonreal-time & $\begin{array}{c}64-512 \mathrm{~Kb} / \mathrm{s} \\
(\mathrm{UBR})\end{array}$ & $256 \mathrm{~Kb} / \mathrm{s}$ & $30-36000$ seconds & 180 seconds & $\begin{array}{c}\text { Remote Login \& Data on } \\
\text { Demand }\end{array}$ \\
\hline 6 & Nonreal-time & $\begin{array}{c}1-10 \mathrm{Mb} / \mathrm{s} \\
(\mathrm{UBR})\end{array}$ & $5 \mathrm{Mb} / \mathrm{s}$ & $30-1200$ seconds & 120 seconds & $\begin{array}{c}\text { File Transfer \& Retrieval } \\
\text { Service }\end{array}$ \\
\hline
\end{tabular}

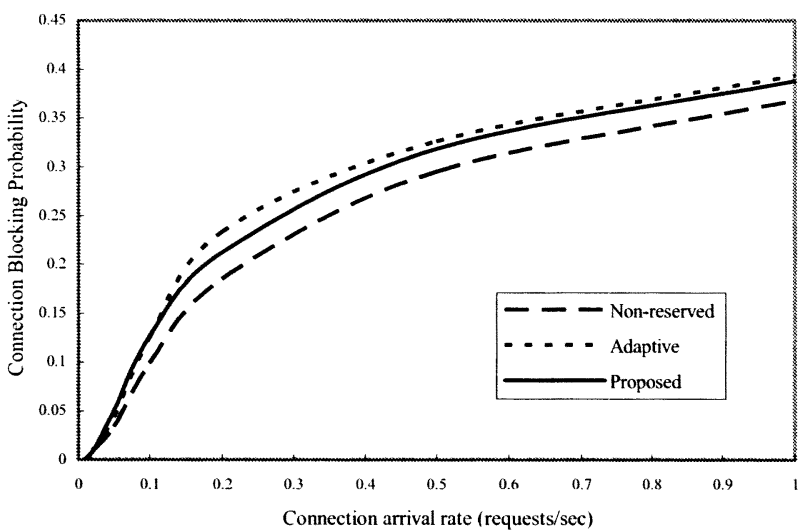

Figure 2. Connection-blocking probability (CBP).

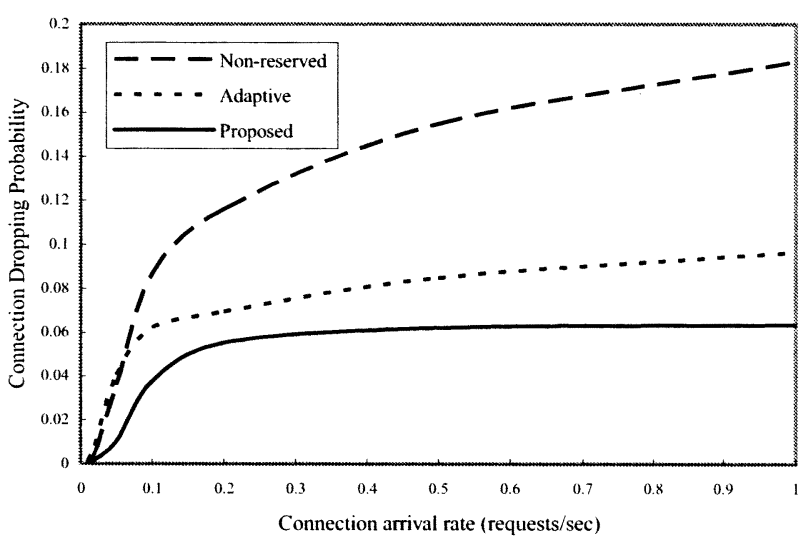

Figure 3. Connection-dropping probability (CDP).

val rate is larger than 0.1 requests/sec, it is evident that the CBPs of non-reserved bandwidth mechanism and our proposed scheme are better than that of the adaptive resource allocation scheme. Figure 3 shows that the CDP of our proposed scheme is better than that of the adaptive resource allocation scheme and non-reserved bandwidth

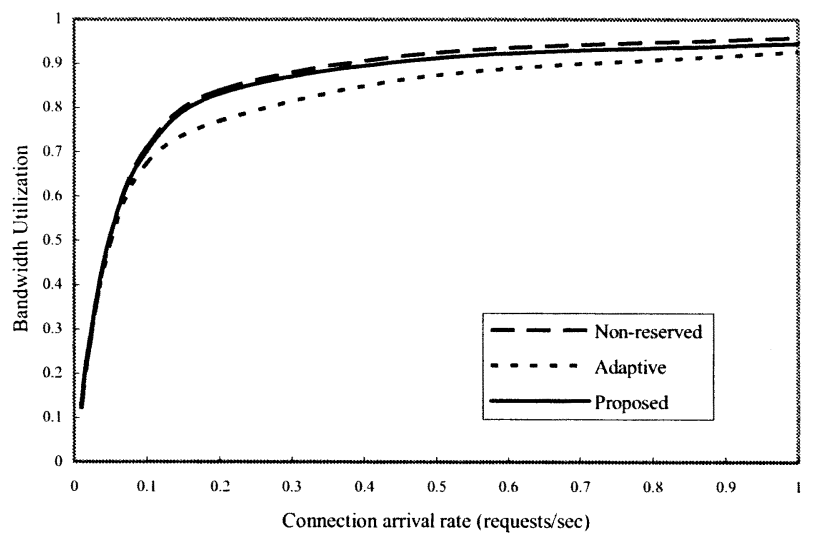

Figure 4. Bandwidth utilization (BU).

mechanism. Furthermore, as the connection arrival rate increases, it is obvious that the proposed scheme results in lower CDP. For example, when the connection arrival rate is 0.5 requests/sec, the proposed scheme is easy to achieve a reduction in percentage of dropped connections from $15 \%$ to $6 \%$, which is compared with the non-reserved bandwidth mechanism. Furthermore, in this case, the proposed scheme achieves the CDP that is approximately $2 \%$ lower than the adaptive resource allocation scheme. The reason for this behavior is that the proposed scheme dynamically adjusts the bandwidth reservation for each BS by using traffic-based reservation strategy, providing more precise bandwidth reservation and efficient call admission control for handoff connections. Therefore, the CDP can be reduced. Reduction of CDP and CBP are contradictory. Hence, we must make a tradeoff between the BU and bandwidth reservation such that the CDP for handoff connections can be reduce, while the CBP for new connections can be maintained at an acceptable level. Via simulations, we show that our proposed scheme reaches these significant objectives.

Figure 4 shows the BU. The non-reserved bandwidth mechanism results in higher BU than adaptive resource allocation scheme and our proposed scheme. This is be- 
cause the non-reserved bandwidth mechanism does not reserve any bandwidth for handoff connections, and thus no bandwidth is wasted. The adaptive resource allocation scheme is suitable for the uniformly traffic conditions. However, when the traffic conditions are bursts, this scheme does not appropriately adjust the reserved bandwidth for each BS. Hence the bandwidth is wasted. In contrast, our proposed scheme adjusts bandwidth reservation by using the attribute-measurement mechanism and trafficbased reservation strategy, leading to handling bursts of traffic conditions efficiently. The amount of reserved bandwidth for each BS is dynamically adjusted, according to the on-line traffic information of each BS. It reserves bandwidth only when necessary, resulting in higher BU. According to the Figure 4, when the connection arrival rate is lower than 0.1 requests $/ \mathrm{sec}$, the BUs of three mechanisms are similar. However, when the connection arrival rate increases, the BU of our proposed scheme is close to that of the non-reserved bandwidth mechanism and superior to that of the adaptive resource allocation scheme.

\section{Conclusion}

The major advantage of this paper is that our proposed scheme is successfully applied to deal with bandwidth reservation problems and provides better QoS guarantees in mobile multimedia wireless networks. In the proposed scheme, the amount of reserved bandwidth for each BS is dynamically adjusted, according to the on-line traffic information of each BS. The simulation results show that the bandwidth reservation scheme using dynamically adaptive techniques improves $\mathrm{CBP}, \mathrm{CDP}$, and BU significantly. The proposed scheme has significantly lower complexity and system overhead than mobility oriented methods. It not only outperforms the pervious scheme, but is also suitable for the next generation mobile communication systems.

\section{References}

[1] L. Huang, S. Kumar, and C.-C. Jay Kuo, "Adaptive Resource Allocation for Multimedia QoS Management in wireless Networks," IEEE Trans. Veh. Technol., vol. 53, no. 2, pp. 547-558, March 2004.

[2] D. A. Levine, I. F. Akyildiz, and M. Naghshineh, "A Resource Estimation and Call Admission Algorithm for Wireless Multimedia Networks Using the Shadow Cluster Concept," IEEE/ACM Trans. Networking, vol. 5, no. 1, pp. 1-12, Feb. 1997.

[3] C. Oliverira, J. B. Kim, and T. Suda, "An Adaptive Bandwidth Reservation Scheme for High-Speed Multimedia Wireless Networks," IEEE . Select. Areas Commun., vol. 16, no. 6, pp. 858-874, Aug. 1998.

[4] J. Ye, J. Hou, and S. Papavassiliou, "A Comprehensive Resource Management Framework for Next Generation Wireless Networks," IEEE Trans. Mobile Computing, vol. 1, no. 4, pp. 249-264, Oct.-Dec. 2002.

[5] L. Badia, M. Zorzi, and A. Gazzini, "A Model for Threshold Comparison Call Admission Control in Third Generation Cellular Systems," in Proc. IEEE Int. Conf. Communications (ICC'03), 2003, pp. 1664-1668.

[6] J. Ye, J. Hou, and S. Papavassiliou, "Integration of Advanced Reservation and Bandwidth Reconfiguration Based Admission Control in Wireless Networks with Multimedia Services," in Proc. IEEE Int. Conf. on Distributed Computing Systems Workshops (ICDCSW'03), 2003. pp. 844-849. 